\title{
A Benchmark Approach to Filtering in Finance
}

\section{Eckhard Platen $^{1}$ and Wolfgang J. Runggaldier ${ }^{2}$}

\author{
September 3, 2004
}

\begin{abstract}
The paper proposes the use of the growth optimal portfolio for pricing and hedging in incomplete markets when there are unobserved factors that have to be filtered. The proposed filtering framework is applicable also in cases when there does not exist an equivalent risk neutral martingale measure. The reduction of the variance of derivative prices for increasing degrees of available information is measured.
\end{abstract}

1991 Mathematics Subject Classification: primary 90A09; secondary 60G99, 62P20. JEL Classification: G10, G13

Key words and phrase: Financial modeling, stochastic filtering, benchmark approach, growth optimal portfolio, fair pricing under partial information.

\footnotetext{
${ }^{1}$ University of Technology Sydney, School of Finance \& Economics and Department of Mathematical Sciences, PO Box 123, Broadway, NSW, 2007, Australia

${ }^{2}$ Universitá degli Studi di Padova, Dipartimento di Matematica Pura ed Applicata, Via Belzoni, 7 I - 35131 Padova, Italy
} 


\section{Introduction}

In financial modeling it is typically the case that, in practice, not all quantities which determine the dynamics of security prices can be fully observed. Some of the factors that characterize the evolution of the market are hidden. For instance, correlations between driving Wiener processes often change quite randomly after certain periods of time. An example is the correlation between the fluctuations of specific and general market risk, see Platen \& Stahl (2003). However, these unobserved factors, correctly calibrated, are essential to reflect the market dynamics that one empirically observes in a financial market model. This leads naturally to a stochastic filtering problem. Given the available information, filter methods can be used to determine the distribution, called the filter distribution, of the unobserved factors. This distribution allows one to compute the expectations of quantities that are dependent on unobserved factors, thereby enabling one to determine such quantities as derivative prices, optimal portfolio strategies and risk measures.

There is a growing literature in the area of filtering in finance. To mention a few recent publications, let us list Elliott \& van der Hoek (1997), Fischer, Platen \& Runggaldier (1999), Elliott, Fischer \& Platen (1999), Fischer \& Platen (1999), Landen (2000), Gombani \& Runggaldier (2001), Frey \& Runggaldier (1999, 2001), Elliott \& Platen (2001), Bhar, Chiarella \& Runggaldier (2002, 2004) and Chiarella, Pasquali \& Runggaldier (2001). These papers provide examples where filter methods have been applied to dynamic asset allocation, interest rate term structure calibration, risk premia estimation, volatility estimation and hedging under partial observation.

A key problem that arises in most filtering applications in finance is the identification of a suitable pricing measure. Results often depend significantly on the assumptions made in choosing such a measure. Furthermore, it has been demonstrated in Heath \& Platen (2002b), Platen (2004b) and Breymann, Kelly \& Platen (2004) that any realistic parsimonious financial market model is unlikely to have an equivalent risk neutral martingale measure. Moreover, in filtering it is obvious that one has to deal with the real world probability measure in order to extract estimates for the hidden factors from observations. It is therefore very important to explore methods based purely on the real world probability measure which allow for consistent filtering under partial information. Applications for such techniques include derivative pricing, portfolio optimization and risk measurement among others.

In this paper we extend the benchmark approach proposed in Platen $(2002,2004 \mathrm{a}$, $2004 \mathrm{~b})$ to filtering. There, as here, the benchmark or numeraire is chosen to be the growth optimal portfolio (GOP). This extends work by Long (1990) and BajeuxBesnainou \& Portait (1997) to the general case where no equivalent risk neutral measure exists. The proposed setup is rich enough to accommodate jump diffusion financial market models with hidden factors. We therefore provide results 
for the case of partial information in an incomplete market framework.

The GOP has the economic interpretation of being the portfolio that maximizes expected logarithmic utility. It is highly relevant in portfolio optimization, see Korn \& Schäl (1999), but also for derivative pricing, as we will see below. By using the GOP as numeraire or benchmark under a given information structure, one naturally obtains a fair derivative price system, where benchmarked derivative prices are martingales. This means that benchmarked derivative prices equal their expected future benchmarked values. The delicate interaction between measures and various information structures is avoided that has to be dealt with if a risk neutral methodology is applied.

In the special case of a complete market with an equivalent risk neutral martingale measure, it will be shown that fair prices coincide with risk neutral prices. Furthermore, for an incomplete market with a minimal equivalent martingale measure in the sense of Föllmer \& Schweizer (1991), it will turn out that prices arising from the use of this measure coincide with fair prices obtained under the benchmark approach. For incomplete jump diffusion markets under partial observation, a much wider range of models is covered in this paper than under traditional approaches.

It will be shown that all portfolios, when expressed in units of the GOP, turn out to be local martingales with respect to the given real world measure and under partial information. The above mentioned difficulties that result from measure transformations under various information structures cannot arise under the proposed fair pricing and major risk management tasks, such as hedging, portfolio optimization and risk measurement, can be performed consistently under the real world measure. Moreover, in cases where no equivalent risk neutral measure exists, the benchmark approach overcomes restrictions arising from the risk neutral methodology, such as those described in Delbaen \& Schachermayer (1995, 1998), for instance.

The paper is structured in the following way. Section 2 summarizes the general filtering methodology for multi-factor jump diffusion models under partial observation. Section 3 describes the proposed filtered benchmark model. The fair pricing of derivatives is then studied in Section 4. This section also quantifies the reduction of variance for derivative prices when additional information is available. Hedging under partial observation is subject of Section 5. 


\section{Filtered Multi-Factor Models}

\section{$2.1 \quad$ Factor Model}

To build a financial market model with a sufficiently rich structure and high computational tractability we introduce a multi-factor model. We also aim to cover market dynamics for which an equivalent risk neutral martingale measure does not exist. This is important for practical reasons, since it has been highlighted in Platen (2004d) that any realistic stock market model is unlikely to have an equivalent risk neutral martingale measure. Detailed analysis of intraday data, such as performed by Breymann, Kelly \& Platen (2004), reveals that there exist natural parameter processes that are only indirectly observable. The correlation processes between the Wiener processes which drive different factors provide a typical example of such hidden quantities. These correlations are extremely important for fund management and derivative pricing.

We consider a multi-factor model with $n \geq 2$ factors, which constitute the vector process

$$
z=\left\{z_{t}=\left(z_{t}^{1}, \ldots, z_{t}^{n}\right)^{\top}, t \in[0, T]\right\}
$$

where $T$ is a fixed time horizon. We assume that not all of the factors are observed. More precisely, only the first $k$ factors are directly observed, while the remaining $n-k$ are not. Later on the unobserved factors will be treated with filtering methods. We refer to $k$ as the degree of available information. Here $k$ is an integer with $1 \leq k<n$. We will assume that its value is fixed for most of this paper. However, in Section 4.3 we discuss the implications of a varying $k$, that is, of a varying degree of information.

For fixed $k$ we consider the following subvectors of $z_{t}$ :

$y_{t}=\left(y_{t}^{1}, \ldots, y_{t}^{k}\right)^{\top}=\left(z_{t}^{1}, \ldots, z_{t}^{k}\right)^{\top} \quad$ and $\quad x_{t}=\left(x_{t}^{1}, \ldots, x_{t}^{n-k}\right)^{\top}=\left(z_{t}^{k+1}, \ldots, z_{t}^{n}\right)^{\top}$,

with $y_{t}$ representing the observed factors and $x_{t}$ the unobserved factors. To be specific, we assume that $y_{t}$ includes the observed security prices as components. These are given by $d+1$ primary security account processes $S^{(0)}, S^{(1)}, \ldots, S^{(d)}$, $d \in\{1,2, \ldots, k-1\}$. We set $y_{t}^{j+1}=S_{t}^{(j)}$ for $j \in\{0,1, \ldots, d\}$ and $t \in[0, T]$. We assume that a primary security account holds only units of one security and that the income or payments accrued from holding this security are always capitalized. In the case of shares, this models the usual ownership of productive units. We consider $S^{(0)}=\left\{S_{t}^{(0)}, t \in[0, T]\right\}$ to be the savings account process, where $S_{t}^{(0)}=$ $\exp \left\{\int_{0}^{t} r_{s} d s\right\}$. This means that we consider the short rate $r_{t}$ to be observable. Furthermore, the dynamics of $y_{t}^{j}$ for $j \in\{1,2, \ldots, d+1\}$ are assumed to be such that these observed quantities always remain nonnegative. Note that the market can be incomplete.

The following setup is relatively complex because we aim to provide a general 
framework covering Markovian jump diffusion market models with hidden factors driven by a finite number of Wiener and Poisson processes. Assume the existence of a filtered probability space $\left(\Omega, \mathcal{A}_{T}, \underline{\mathcal{A}}, P\right)$, where $\underline{\mathcal{A}}=\left(\mathcal{A}_{t}\right)_{t \in[0, T]}$ is a filtration to which all the processes considered are adapted. We assume that the observed and unobserved factors satisfy the system of stochastic differential equations (SDEs)

$$
\begin{aligned}
& d x_{t}=a_{t}\left(z_{t}\right) d t+b_{t}\left(z_{t}\right) d w_{t}+g_{t-}\left(z_{t-}\right) d m_{t} \\
& d y_{t}=A_{t}\left(z_{t}\right) d t+B_{t}\left(y_{t}\right) d v_{t}+G_{t-}\left(y_{t-}\right) d N_{t}
\end{aligned}
$$

for $t \in[0, T]$, with given vector of initial values $z_{0}=\left(y_{0}^{1}, \ldots, y_{0}^{k}, x_{0}^{1}, \ldots, x_{0}^{n-k}\right)^{\top}$. Here

$$
w=\left\{w_{t}=\left(w_{t}^{1}, \ldots, w_{t}^{k}, w_{t}^{k+1}, \ldots, w_{t}^{n}\right)^{\top}, t \in[0, T]\right\}
$$

is an $n$-dimensional $(\underline{\mathcal{A}}, P)$-Wiener process and

$$
v_{t}=\left(w_{t}^{1}, \ldots, w_{t}^{k}\right)^{\top}
$$

is the subvector of its first $k$ components. The process $m=\left\{m_{t}=\left(m_{t}^{1}, \ldots, m_{t}^{k}\right.\right.$, $\left.\left.m_{t}^{k+1}, \ldots, m_{t}^{n}\right)^{\top}, t \in[0, T]\right\}$ is an $n$-dimensional $(\underline{\mathcal{A}}, P)$-jump martingale defined as follows. Consider $n$ counting processes $N^{1}, \ldots, N^{n}$ with no common jumps. These are characterized by the corresponding vector of intensities $\lambda_{t}\left(z_{t}\right)=$ $\left(\lambda_{t}^{1}\left(z_{t}\right), \ldots, \lambda_{t}^{n}\left(z_{t}\right)\right)^{\top}$, where

$$
\lambda_{t}^{i}\left(z_{t}\right)=\tilde{\lambda}_{t}^{i}\left(y_{t}\right)
$$

for $t \in[0, T]$ and $i \in\{1,2, \ldots, k\}$. This means that we assume, without loss of generality, that the jump intensities of the first $k$ counting processes are observed. The $i$ th $(\underline{\mathcal{A}}, P)$-jump martingale is then defined by the stochastic differential

$$
d m_{t}^{i}=d N_{t}^{i}-\lambda_{t}^{i}\left(z_{t-}\right) d t
$$

for $t \in[0, T]$ and $i \in\{1,2, \ldots, n\}$. In $(2.3)$

$$
N_{t}=\left(N_{t}^{1}, \ldots, N_{t}^{k}\right)^{\top}
$$

denotes the vector of the first $k$ counting processes at time $t \in[0, T]$. Concerning the coefficients in the $\operatorname{SDE}(2.3)$, we assume that the vectors $a_{t}\left(z_{t}\right), A_{t}\left(z_{t}\right), \lambda_{t}\left(z_{t}\right)$ and the matrices $b_{t}\left(z_{t}\right), B_{t}\left(y_{t}\right), g_{t}\left(z_{t}\right)$ and $G_{t}\left(y_{t}\right)$ are such that a unique strong solution of (2.3), which does not explode until time $T$, exists, see Protter (1990).

As mentioned before, the components $y_{t}^{1}, \ldots, y_{t}^{d+1}$ are assumed to be nonnegative for all $t \in[0, T]$. We shall also assume that the $k \times k$-matrix $B_{t}\left(y_{t}\right)$ is invertible for all $t \in[0, T]$. We assume further that $A_{t}\left(z_{t}\right)$ and $B_{t}\left(y_{t}\right)$ in (2.3) are such that

$$
\int_{0}^{T} E\left(\left|A_{t}\left(z_{t}\right)\right|\right) d t<\infty \text { and } \int_{0}^{T}\left|B_{t}\left(y_{t}\right) B_{t}\left(y_{t}\right)^{\top}\right| d t<\infty
$$

$P$-a.s. Finally, $g_{t}\left(z_{t}\right)$ may be any bounded function and the $k \times k$-matrix $G_{t}\left(y_{t}\right)$ is assumed to be an invertible matrix valued function of $y_{t}$ for each $t \in[0, T]$. 
The latter assumption implies that, since there are no common jumps among the components of $N_{t}$, by observing a jump of $y_{t}$ we can establish which of the processes $N^{i}, i \in\{1,2, \ldots, k\}$, has jumped.

In addition to the filtration $\underline{\mathcal{A}}$, which represents complete information, we will also consider the subfiltration

$$
\tilde{\mathcal{A}}^{k}=\left(\tilde{\mathcal{A}}_{t}^{k}\right)_{t \in[0, T]} \subseteq \underline{\mathcal{A}},
$$

where $\tilde{\mathcal{A}}_{t}^{k}=\sigma\left\{y_{s}=\left(z_{s}^{1}, \ldots, z_{s}^{k}\right)^{\top}, s \leq t\right\}$ represents the observed information up to time $t \in[0, T]$. Thus $\tilde{\mathcal{A}}^{k}$ provides the structure of the information actually available in the market.

We shall be interested in the conditional distribution of $x_{t}$ given $\tilde{\mathcal{A}}_{t}^{k}$, which we call, in accordance with standard terminology, the filter distribution at time $t \in[0, T]$. There exist general filter equations for the dynamics described by the SDEs given in (2.3), see Liptser \& Shiryaev (1977). It turns out in our case that these filter equations are SDEs which govern the conditional expectations, with respect to $\tilde{\mathcal{A}}_{t}^{k}$, of integrable functions of the unobserved factors $x_{t}$. Notice, in particular, that $\exp \left\{\imath \nu x_{t}\right\}$ is, for given $\nu \in \Re^{n-k}$ and with $\imath$ denoting the imaginary unit, a bounded and thus integrable function of $x_{t}$. Its conditional expectation with respect to $\tilde{\mathcal{A}}_{t}^{k}$ is the conditional characteristic function of the distribution of $x_{t}$ given $\tilde{\mathcal{A}}_{t}^{k}$. This completely characterizes the entire filter distribution. Considering conditional expectations of integrable functions of $x_{t}$ is thus not too restrictive for the identification of filter distributions.

The general case of filter equations is beyond the scope of this paper. These are, for instance, considered in Liptser \& Shiryaev (1977). To keep the filter reasonably tractable we assume that the SDEs given in (2.3) are such that the corresponding filter distributions admit a representation of the form

$$
F_{z_{t}^{k+1}, \ldots, z_{t}^{n}}\left(z_{1}, \ldots, z_{n-k} \mid \zeta_{t}^{1}, \ldots, \zeta_{t}^{q}\right)=P\left(z_{t}^{k+1} \leq z_{1}, \ldots, z_{t}^{n} \leq z_{n-k} \mid \tilde{\mathcal{A}}_{t}^{k}\right)
$$

for all $\left(z_{1}, \ldots, z_{n-k}\right)^{\top} \in \Re^{n-k}$ and $t \in[0, T]$. This means that we have a finitedimensional filter, characterized by the filter state process

$$
\zeta=\left\{\zeta_{t}=\left(\zeta_{t}^{1}, \ldots, \zeta_{t}^{q}\right)^{\top}, t \in[0, T]\right\}
$$

which is an $\tilde{\mathcal{A}}^{k}$-adapted process with a certain finite dimension $q \geq 1$. We shall denote by $\tilde{z}_{t}^{k}$ the resulting $(k+q)$-vector of observables

$$
\tilde{z}_{t}^{k}=\left(y_{t}^{1}, \ldots, y_{t}^{k}, \zeta_{t}^{1}, \ldots, \zeta_{t}^{q}\right)^{\top},
$$

which consists of the $k$ observed factors and the $q$ components of the filter state process. Furthermore, we assume that the filter state $\zeta_{t}$ satisfies an SDE of the form

$$
d \zeta_{t}=C_{t}\left(\tilde{z}_{t}^{k}\right) d t+D_{t-}\left(\tilde{z}_{t-}^{k}\right) d y_{t}
$$


for $t \in[0, T]$, with $C_{t}(\cdot)$ denoting a $q$-vector valued function and $D_{t}(\cdot)$ a $(q \times k)$ matrix valued function.

There are various models of the type (2.3) that admit a finite-dimensional filter with $\zeta_{t}$ subject to an equation of the form (2.14). In the following two subsections we recall two classical such models. These are the conditionally Gaussian model, which leads to a generalized Kalman filter and the finite-state jump model for $x$, which is related to hidden Markov chain filters. Various combinations of these models have finite-dimensional filters and can be readily applied in finance, as demonstrated in the literature mentioned in the introduction.

\section{Example 2.1 : $\quad$ Conditionally Gaussian Filter Model}

Assume in the system of SDEs $(2.3)$ that the functions $a_{t}(\cdot)$ and $A_{t}(\cdot)$ are linear in the factors, that $b_{t}\left(z_{t}\right)=b_{t}$ is deterministic and that $g_{t}\left(z_{t}\right)=G_{t}\left(y_{t}\right)=0$. This means that (2.3) takes the form

$$
\begin{aligned}
d x_{t} & =\left[a_{t}^{0}+a_{t}^{1} x_{t}+a_{t}^{2} y_{t}\right] d t+b_{t} d w_{t} \\
d y_{t} & =\left[A_{t}^{0}+A_{t}^{1} x_{t}+A_{t}^{2} y_{t}\right] d t+B_{t}\left(y_{t}\right) d v_{t}
\end{aligned}
$$

for $t \in[0, T]$, with given deterministic initial values $x_{0}$ and $y_{0}$. Here $a_{t}^{0}$ and $A_{t}^{0}$ are column vectors of dimensions $(n-k)$ and $k$, respectively, and $a_{t}^{1}, a_{t}^{2}, b_{t}$, $A_{t}^{1}, A_{t}^{2}$ and $B_{t}\left(y_{t}\right)$ are matrices with appropriate dimensions. Recall that $w$ is an $n$-dimensional $(\underline{\mathcal{A}}, P)$-Wiener process and that $v$ is the vector of its first $k$ components.

In this case the filter distribution is a Gaussian distribution with vector mean $\mu_{t}=\left(\mu_{t}^{1}, \ldots, \mu_{t}^{n-k}\right)^{\top}$, where

$$
\mu_{t}^{i}=E\left(x_{t}^{i} \mid \tilde{\mathcal{A}}_{t}^{k}\right)
$$

for $t \in[0, T]$. Its covariance matrix is $c_{t}=\left[c_{t}^{\ell, i}\right]_{\ell, i \in\{1,2, \ldots, n-k\}}$, where

$$
c_{t}^{\ell, i}=E\left(\left(x_{t}^{\ell}-\mu_{t}^{\ell}\right)\left(x_{t}^{i}-\mu_{t}^{i}\right) \mid \tilde{\mathcal{A}}_{t}^{k}\right)
$$

for $t \in[0, T]$. The dependence of $\mu_{t}$ and $c_{t}$ on $k$ is, for simplicity, suppressed in our notation. The above filter can be obtained from a generalization of the well-known Kalman filter, see Liptser \& Shiryaev (1977), whose filter distribution is characterized by

$$
\begin{aligned}
& d \mu_{t}= {\left[a_{t}^{0}+a_{t}^{1} \mu_{t}+a_{t}^{2} y_{t}\right] d t+\left[\bar{b}_{t} B_{t}\left(y_{t}\right)^{\top}+c_{t}\left(A_{t}^{1}\right)^{\top}\right]\left(B_{t}\left(y_{t}\right) B_{t}\left(y_{t}\right)^{\top}\right)^{-1} } \\
& \cdot\left[d y_{t}-\left(A_{t}^{0}+A_{t}^{1} \mu_{t}+A_{t}^{2} y_{t}\right) d t\right] \\
& d c_{t}=\left(a_{t}^{1} c_{t}+c_{t}\left(a_{t}^{1}\right)^{\top}+\left(b_{t} b_{t}^{\top}\right)\right. \\
&\left.-\left[\bar{b}_{t} B_{t}\left(y_{t}\right)^{\top}+c_{t}\left(A_{t}^{1}\right)^{\top}\right]\left(B_{t}\left(y_{t}\right) B_{t}\left(y_{t}\right)^{\top}\right)^{-1}\left[\bar{b}_{t} B_{t}\left(y_{t}\right)^{\top}+c_{t}\left(A_{t}^{1}\right)^{\top}\right]^{\top}\right) d t,
\end{aligned}
$$


where $\bar{b}_{t}$ is the $(k \times k)$-upper left block of $b_{t}, t \in[0, T]$. We recall that $B_{t}\left(y_{t}\right)$ is assumed to be invertible.

Notice that the computation of $c_{t}$ is contingent upon the knowledge of the coefficients in the second equation of (2.18). These coefficients are given deterministic functions of time, except for $B_{t}\left(y_{t}\right)$, which also depends on observed factors. Although $c_{t}$ is defined as a conditional expectation for $t \in[0, T]$, it follows from (2.18) that if $B_{t}\left(y_{t}\right)$ does not depend on the observable factors $y_{t}$, then $c_{t}$ can be computed off-line. In general, the value of $B_{t}\left(y_{t}\right)$ becomes known only at time $t$. However, this is sufficient to determine the solution of (2.18) at time $t$. The model (2.15) is in fact of the type of a conditionally Gaussian filter model, where the filter-state process $\zeta$ is given by the vector process $\mu=\left\{\mu_{t}, t \in[0, T]\right\}$ and the upper triangular array of elements of the matrix process $c=\left\{c_{t}, t \in[0, T]\right\}$. The dimension of the filter-state process is then $q=(n-k) \frac{[3+(n-k)]}{2}$. Note by (2.17) that the matrix $c_{t}$ is symmetric. Obviously, in the case when $B_{t}\left(y_{t}\right)$ does not depend on $y_{t}$, we have a Gaussian filter model.

\section{Example 2.2 : Finite-State Jump Model}

Here we assume that the unobserved factors form a continuous time, $(n-k)$ dimensional jump process $x=\left\{x_{t}=\left(x_{t}^{1}, \ldots, x_{t}^{n-k}\right)^{\top}, t \in[0, T]\right\}$, which can take a finite number $M$ of values. More precisely, given an appropriate time $t$ and $z_{t^{-}}$ dependent matrix $g_{t}\left(z_{t}\right)$ and an intensity vector $\lambda_{t}\left(z_{t}\right)=\left(\lambda_{t}^{1}\left(z_{t}\right), \ldots, \lambda_{t}^{n}\left(z_{t}\right)\right)^{\top}$ for the vector counting process $\bar{N}=\left\{\bar{N}_{t}=\left(N_{t}^{1}, \ldots, N_{t}^{n}\right)^{\top}, t \in[0, T]\right\}$, we consider the particular case of the model equations $(2.3)$, where in the $x_{t}$-dynamics $a_{t}\left(z_{t}\right)=$ $g_{t}\left(z_{t}\right) \lambda_{t}\left(z_{t}\right)$ and $b_{t}\left(z_{t}\right)=0$. Thus, by (2.3) and (2.7) we have

$$
d x_{t}=g_{t-}\left(z_{t-}\right) d \bar{N}_{t}
$$

for $t \in[0, T]$. Notice that the process $x$ of unobserved factors is a pure jump process and is therefore piecewise constant. On the other hand, we assume that the vector $y_{t}$ satisfies the second equation in $(2.3)$ with $G_{t}\left(y_{t}\right)=0$. This means that the process of observed factors $y$ is only perturbed by continuous noise and does not jump.

In this example, the filter distribution is completely characterized by the vector of conditional probabilities $p_{t}=\left(p_{t}^{1}, \ldots, p_{t}^{M}\right)^{\top}$, where $M$ is the number of possible states $\eta^{1}, \ldots, \eta^{M}$ of the vector $x_{t}$ and

$$
p_{t}^{j}=P\left(x_{t}=\eta^{j} \mid \tilde{\mathcal{A}}_{t}^{k}\right)
$$

for $t \in[0, T]$ and $j \in\{1,2, \ldots, M\}$. Let $\bar{a}_{t}^{i, j}\left(y, \eta^{h}\right)$ denote the transition kernel for $x$ to jump from state $i$ to state $j$, given that $y_{t}=y$ and $x_{t}=\eta^{h}$ at time $t$, see Liptser \& Shiryaev (1977). The components of the vector $p_{t}$ satisfy the following 
dynamics

$$
\begin{aligned}
d p_{t}^{j}=\left(\tilde{a}_{t}\left(y_{t}, p_{t}\right)^{\top} p_{t}\right)^{j} d t+p_{t}^{j}\left[A_{t}\left(y_{t}, \eta^{j}\right)-\tilde{A}_{t}\left(y_{t}, p_{t}\right)\right]\left(B_{t}\left(y_{t}\right) B_{t}\left(y_{t}\right)^{\top}\right)^{-1} \\
\cdot\left[d y_{t}-\tilde{A}_{t}\left(y_{t}, p_{t}\right) d t\right]
\end{aligned}
$$

where

$$
\begin{aligned}
\left(\tilde{a}_{t}\left(y_{t}, p_{t}\right)^{\top} p_{t}\right)^{j} & =\sum_{i=1}^{M}\left(\sum_{h=1}^{M} \bar{a}_{t}^{i, j}\left(y_{t}, \eta^{h}\right) p_{t}^{h}\right) p_{t}^{i} \\
A_{t}\left(y_{t}, \eta^{j}\right) & =\left.A_{t}\left(y_{t}, x_{t}\right)\right|_{x_{t}=\eta^{j}} \\
\tilde{A}_{t}\left(y_{t}, p_{t}\right) & =\sum_{j=1}^{M} A_{t}\left(y_{t}, \eta^{j}\right) p_{t}^{j}
\end{aligned}
$$

for $t \in[0, T]$ and $j \in\{1,2, \ldots, M\}$. The filter state process $\zeta=\left\{\zeta_{t}=\left(\zeta_{t}^{1} \ldots, \zeta_{t}^{q}\right)^{\top}\right.$, $t \in[0, T]\}$ for the finite state jump model is thus given by the vector process $p=\left\{p_{t}=\left(p_{t}^{1}, \ldots, p_{t}^{q}\right)^{\top}, t \in[0, T]\right\}$ with $q=M-1$. Since the probabilities add up to one, we need only $M-1$ probabilities to characterize the filter.

\subsection{Markovian Representation}

As in the two previous examples, in general, we have to deal in our filter setup with the quantity $E\left(A_{t}\left(z_{t}\right) \mid \tilde{\mathcal{A}}_{t}^{k}\right)$, assuming that it exists. This is the conditional expectation, with respect to the filter distribution at time $t$ for the unobserved factors, of the coefficient $A_{t}\left(z_{t}\right)=A_{t}\left(y_{t}^{1}, \ldots, y_{t}^{k}, x_{t}^{1}, \ldots, x_{t}^{n-k}\right)$ that appears in (2.3). Since the filter is characterized by the filter state process $\zeta$, we obtain the representation

$$
\tilde{A}_{t}\left(\tilde{z}_{t}^{k}\right)=E\left(A_{t}\left(z_{t}\right) \mid \tilde{\mathcal{A}}_{t}^{k}\right)
$$

for this conditional expectation, where the vector $\tilde{z}_{t}^{k}$ is as defined in (2.13). Note in our financial market context that we deal with conditional expectations under the real world probability measure. This is an essential observation for understanding the theoretical and practical benefits of the benchmark approach that we pursue later on.

Notice that, in the case of the conditionally Gaussian model in Example 2.1, the expression $\tilde{A}_{t}\left(\tilde{z}_{t}^{k}\right)$ takes the particular form

$$
\tilde{A}_{t}\left(\tilde{z}_{t}^{k}\right)=A_{t}^{0}+A_{t}^{1} \mu_{t}+A_{t}^{2} y_{t} .
$$

Furthermore, for the finite-state jump model of Example 2.2, $\tilde{\mathcal{A}}_{t}\left(\tilde{z}_{t}^{k}\right)$ can be represented as

$$
\tilde{A}_{t}\left(\tilde{z}_{t}^{k}\right)=\tilde{A}_{t}\left(y_{t}, p_{t}\right)=\sum_{j=1}^{M} A_{t}\left(y_{t}, \eta^{j}\right) p_{t}^{j}
$$


for $t \in[0, T]$, see $(2.22)$.

In Appendix A we prove the following generalization of Theorem 7.12 in Liptser \& Shiryaev (1977), which provides an important representation of the SDE for the observed factors.

Proposition 2.3 Let $A_{t}\left(z_{t}\right)$ and the invertible matrix $B_{t}\left(y_{t}\right)$ in (2.3) satisfy (2.9). Then there exists a $k$-dimensional $\tilde{\mathcal{A}}^{k}$-adapted Wiener process $\tilde{v}=\left\{\tilde{v}_{t}, t \in\right.$ $[0, T]\}$, such that the process $y=\left\{y_{t}, t \in[0, T]\right\}$ of observed factors in (2.3) satisfies the $S D E$

$$
d y_{t}=\tilde{A}_{t}\left(\tilde{z}_{t}^{k}\right) d t+B_{t}\left(y_{t}\right) d \tilde{v}_{t}+G_{t-}\left(y_{t-}\right) d N_{t}
$$

with $\tilde{A}_{t}\left(\tilde{z}_{t}^{k}\right)$ as in $(2.23)$.

Instead of the original factors $z_{t}=\left(y_{t}^{1}, \ldots, y_{t}^{k}, x_{t}^{1}, \ldots, x_{t}^{n-k}\right)^{\top}=\left(z_{t}^{1}, \ldots, z_{t}^{n}\right)^{\top}$, where $x_{t}=\left(x_{t}^{1}, \ldots, x_{t}^{n-k}\right)^{\top}$ is unobserved, we may now base our analysis on the components of the vector $\tilde{z}_{t}^{k}=\left(y_{t}^{1}, \ldots, y_{t}^{k}, \zeta_{t}^{1}, \ldots, \zeta_{t}^{q}\right)^{\top}$, see $(2.13)$, which are all observed. Just as was the case with $z=\left\{z_{t}, t \in[0, T]\right\}$, the vector process $\tilde{z}^{k}=\left\{\tilde{z}_{t}^{k}, t \in[0, T]\right\}$ also has Markovian dynamics. In fact, substituting (2.26) into (2.14), we obtain

$$
\begin{aligned}
d \zeta_{t} & =\left[C_{t}\left(\tilde{z}_{t}^{k}\right)+D_{t}\left(\tilde{z}_{t}^{k}\right) \tilde{A}_{t}\left(\tilde{z}_{t}^{k}\right)\right] d t+D_{t}\left(\tilde{z}_{t}^{k}\right) B_{t}\left(y_{t}\right) d \tilde{v}_{t}+D_{t-}\left(\tilde{z}_{t-}^{k}\right) G_{t-}\left(y_{t-}\right) d N_{t} \\
& =\tilde{C}_{t}\left(\tilde{z}_{t}^{k}\right) d t+\tilde{D}_{t}\left(\tilde{z}_{t}^{k}\right) d \tilde{v}_{t}+\tilde{G}_{t-}\left(\tilde{z}_{t-}^{k}\right) d N_{t}
\end{aligned}
$$

where the vector $\tilde{C}_{t}\left(\tilde{z}_{t}^{k}\right)$ and the matrices $\tilde{D}_{t}\left(\tilde{z}_{t}^{k}\right)$ and $\tilde{G}_{t}\left(\tilde{z}_{t}^{k}\right)$ have been defined implicitly for compact notation.

From equations (2.26) and (2.27) we immediately obtain the following result, which indicates that the process $\tilde{z}^{k}=\left\{\tilde{z}_{t}^{k}, t \in[0, T]\right\}$ is Markovian.

Corollary 2.4 The dynamics of the vector $\tilde{z}_{t}^{k}=\left(y_{t}, \zeta_{t}\right)^{\top}$ can be expressed by the system of SDEs

$$
\begin{aligned}
& d y_{t}=\tilde{A}_{t}\left(\tilde{z}_{t}^{k}\right) d t+B_{t}\left(y_{t}\right) d \tilde{v}_{t}+G_{t-}\left(y_{t-}\right) d N_{t} \\
& d \zeta_{t}=\tilde{C}_{t}\left(\tilde{z}_{t}^{k}\right) d t+\tilde{D}_{t}\left(\tilde{z}_{t}^{k}\right) d \tilde{v}_{t}+\tilde{G}_{t-}\left(\tilde{z}_{t-}^{k}\right) d N_{t} .
\end{aligned}
$$

Due to the existence of Markovian filter dynamics, our original Markovian factor model, given by (2.3), can be projected onto a Markovian model which features only observed quantities. Here the driving observable noise $\tilde{v}$ is an $\left(\tilde{\mathcal{A}}^{k}, P\right)$ Wiener process and the observable counting process $N$ is generated by the first $k$ components $N^{1}, N^{2}, \ldots, N^{k}$ of the $n$ counting processes. 
For efficient notation, we express the system of SDEs for the vector of observables $\tilde{z}_{t}^{k}=\bar{z}_{t}=\left(\bar{z}_{t}^{1}, \bar{z}_{t}^{2}, \ldots, \bar{z}_{t}^{k+q}\right)^{\top}$ as

$$
\begin{aligned}
d \bar{z}_{t}^{\ell}= & \alpha^{\ell}\left(t, \bar{z}_{t}^{1}, \bar{z}_{t}^{2}, \ldots, \bar{z}_{t}^{k+q}\right) d t+\sum_{r=1}^{k} \beta^{\ell, r}\left(t, \bar{z}_{t}^{1}, \bar{z}_{t}^{2}, \ldots, \bar{z}_{t}^{k+q}\right) d \tilde{v}_{t}^{r} \\
& +\sum_{r=1}^{k} \gamma^{\ell, r}\left(t-, \bar{z}_{t-}^{1}, \bar{z}_{t-}^{2}, \ldots, \bar{z}_{t-}^{k+q}\right) d N_{t}^{r}
\end{aligned}
$$

for $t \in[0, T]$ and $\ell \in\{1,2, \ldots, k+q\}$. The functions $\alpha^{\ell}, \beta^{\ell, r}$ and $\gamma^{\ell, r}$ follow directly from $\tilde{A}, B, G, \tilde{C}, \tilde{D}$ and $\tilde{G}$, appearing in (2.28). As an immediate consequence of the Markovianity of $\tilde{z}^{k}=\bar{z}$, as well as property (2.11), we obtain the following result.

Corollary 2.5 Suppose for $t \in[0, T]$ that $E\left(u\left(t, z_{t}\right) \mid \tilde{\mathcal{A}}_{t}^{k}\right)<\infty$ for a given function $u:[0, T] \times \Re^{n} \rightarrow \Re$ and $k \in\{1,2, \ldots, n-1\}$. This Expectation can then be expressed as

$$
E\left(u\left(t, z_{t}\right) \mid \tilde{\mathcal{A}}_{t}^{k}\right)=\tilde{u}^{k}\left(t, \tilde{z}_{t}^{k}\right)=\tilde{u}^{k}\left(t, \bar{z}_{t}\right)
$$

for a suitable function $\tilde{u}^{k}:[0, T] \times \Re^{k+q} \rightarrow \Re$.

Relation (2.30) in Corollary 2.5 is of significant practical importance, in particular, for contingent claim pricing, as we shall see later on.

\section{Benchmark Model}

\subsection{Primary Security Accounts and Portfolios}

Recall from Section 2.1 that in our Markovian jump-diffusion market model with observable and hidden factors we have $d+1$ primary security account processes $S^{(0)}, \ldots, S^{(d)}, d<k$, all of which are observable. This means that the vector process $S=\left\{S_{t}=\left(S_{t}^{(0)}, \ldots, S_{t}^{(d)}\right)^{\top}, t \in[0, T]\right\}$ is $\tilde{\mathcal{A}}^{k}$-adapted. In Section 2.1 we set

$$
y_{t}^{j}=\bar{z}_{t}^{j}=S_{t}^{(j-1)}
$$

for $j \in\{1,2, \ldots, d+1\}$ and $t \in[0, T]$.

Since the $d+1$ primary security account processes coincide with the observable factors $y^{1}, \ldots, y^{d+1}$, we can write their dynamics in a form corresponding to (2.29). To this effect, by analogy to (2.7), let

$$
d \tilde{m}_{t}^{i}=\frac{1}{\sqrt{\tilde{\lambda}_{t-}^{i}\left(\bar{z}_{t-}\right)}}\left(d N_{t}^{i}-\tilde{\lambda}_{t-}^{i}\left(\bar{z}_{t-}\right) d t\right)
$$


for $i \in\{1,2, \ldots, k\}$ be the normalized compensated $i$ th $\left(\tilde{\mathcal{A}}^{k}, P\right)$-jump martingale relative to the filtration $\tilde{\mathcal{A}}^{k}$. Here, with some abuse of notation, we have denoted by $\tilde{\lambda}_{t}^{i}\left(\bar{z}_{t}\right)$ the compensating jump intensity for $N^{i}$ with respect to $\tilde{\mathcal{A}}^{k}$. For simplicity of notation, in what follows we shall often use $\bar{z}_{t}$ for $\tilde{z}_{t}^{k}$, see (2.29). Let us now rewrite (2.29) more concisely in vector form as

$$
d \bar{z}_{t}=\bar{\alpha}\left(t, \bar{z}_{t}\right) d t+\beta\left(t, \bar{z}_{t}\right) d \tilde{v}_{t}+\gamma\left(t-, \bar{z}_{t-}\right) \sqrt{\tilde{\lambda}_{t-}\left(\bar{z}_{t-}\right)} d \tilde{m}_{t}
$$

with

$$
\bar{\alpha}\left(t, \bar{z}_{t}\right)=\alpha\left(t, \bar{z}_{t}\right)+\gamma\left(t-, \bar{z}_{t-}\right) \sqrt{\tilde{\lambda}_{t-}\left(\bar{z}_{t-}\right)}
$$

where $\tilde{m}_{t}=\left(\tilde{m}_{t}^{1}, \ldots, \tilde{m}_{t}^{k}\right)^{\top}$ and $\sqrt{\tilde{\lambda}_{t}}=\left(\sqrt{\tilde{\lambda}_{t}^{1}}, \ldots, \sqrt{\tilde{\lambda}_{t}^{k}}\right)^{\top}$. Here $\alpha\left(t, \bar{z}_{t}\right)$ is a $(k+q)$-column vector and $\beta\left(t, \bar{z}_{t}\right)$ as well as $\gamma\left(t, \bar{z}_{t}\right)$ are $((k+q) \times k)$-matrices.

Since we have assumed that the number $d$ of risky securities is strictly less than the degree $k$ of available information, $d<k$, the primary security accounts do not necessarily span the entire observable uncertainty of the market. Think, for instance, of asset price models which incorporate stochastic volatility, where the volatilities are driven by stochastic processes that are independent from those driving directly the fluctuations of the asset prices. It is therefore reasonable to assume that among the driving random processes $\tilde{v}^{i}$ and $\tilde{m}^{\ell}, i, \ell \in\{1,2, \ldots, k\}$, those that directly drive the fluctuations of the risky primary security accounts $S_{t}^{(j)}, j \in\{1,2, \ldots, d\}$, are exactly $d$ in number. We shall thus assume that for any $j \in\{0,1, \ldots, d\}$ the dynamics of the $j$ th primary security account is given by the SDE

$$
d S_{t}^{(j)}=\bar{\alpha}^{j}\left(t, \bar{z}_{t}\right) d t+\sum_{i=1}^{h_{1}} \beta^{j, i}\left(t, \bar{z}_{t}\right) d \tilde{v}_{t}^{i}+\sum_{\ell=1}^{h_{2}} \gamma^{j, \ell}\left(t-, \bar{z}_{t-}\right) \sqrt{\tilde{\lambda}_{t-}^{\ell}\left(\bar{z}_{t-}\right)} d \tilde{m}_{t}^{\ell}
$$

for $t \in[0, T]$, where $h_{1}+h_{2}=d$.

We assume that all model specifications are such that a unique strong solution of the system of SDEs (3.2) exists, see Protter (1990). For efficient and more transparent notation we now rewrite the SDE (3.4) in the form

$$
d S_{t}^{(j)}=S_{t-}^{(j)}\left(r_{t} d t+\sum_{i=1}^{h_{1}} b_{t}^{j, i}\left(d \tilde{v}_{t}^{i}+\theta_{t}^{i} d t\right)+\sum_{\ell=h_{1}+1}^{d} b_{t-}^{j, \ell}\left(d \tilde{m}_{t}^{\ell-h_{1}}+\theta_{t-}^{\ell} d t\right)\right)
$$

for $t \in[0, T]$, with $S_{t}^{(j)}>0$ and $j \in\{0,1, \ldots, d\}$. Here we set $S_{0}^{(0)}=1$ and $b_{t}^{0, i}=0$ for $t \in[0, T]$ and $i \in\{0,1, \ldots, d\}$, where $r_{t}$ is again the short rate. For the generalized volatility $b_{t}^{j, i}$ in (3.5), if $i \in\left\{1,2, \ldots, h_{1}\right\}$, then

$$
b_{t}^{j, i}=\frac{\beta^{j, i}\left(t, \bar{z}_{t}\right)}{S_{t}^{(j)}}
$$


is a volatility and if $i \in\left\{h_{1}+1, \ldots, d\right\}$, then

$$
b_{t-}^{j, i}=\frac{\gamma^{j, i-h_{1}}\left(t-, \bar{z}_{t-}\right) \sqrt{\tilde{\lambda}_{t-}^{i-h_{1}}\left(\bar{z}_{t-}\right)}}{S_{t-}^{(j)}}
$$

is a jump coefficient for $t \in[0, T]$ and $j \in\{1,2, \ldots, d\}$. We assume that the generalized volatility matrix $b_{t}=\left[b_{t}^{j, i}\right]_{j, i=1}^{d}$ is invertible for all $t \in[0, T]$. This allows us to write the market price for risk vector $\theta_{t}=\left(\theta_{t}^{1}, \ldots, \theta_{t}^{d}\right)^{\top}$ in the form

$$
\theta_{t}=b_{t}^{-1}\left[a_{t}-r_{t} \mathbf{1}\right]
$$

for $t \in[0, T]$. Here $\mathbf{1}=(1, \ldots, 1)^{\top}$ and $a_{t}=\left(a_{t}^{1}, \ldots, a_{t}^{d}\right)^{\top}$ is the appreciation rate vector with

$$
a_{t}^{j}=\frac{\bar{\alpha}^{j}\left(t, \bar{z}_{t}\right)}{S_{t}^{(j)}}
$$

for $t \in[0, T]$ and $j \in\{1,2, \ldots, d\}$. Notice that in the dynamics (3.5) all the coefficients can be determined on the basis of the observables $\bar{z}_{t}$. The interest rate $r_{t}$ was in fact observable with $y_{t}^{1}$ and, by (3.6)-(3.9), the generalized volatility matrix $b_{t}$ and the market price for risk vector $\theta_{t}$ are functions of observables.

Let us form portfolios of primary security accounts. We say that an $\tilde{\mathcal{A}}^{k}$-predictable stochastic process $\delta=\left\{\delta_{t}=\left(\delta_{t}^{0}, \ldots, \delta_{t}^{d}\right)^{\top}, t \in[0, T]\right\}$ is a self-financing strategy, if $\delta$ is $S$-integrable, see Protter (1990), and the corresponding portfolio value

$$
V_{\delta}(t)=\sum_{j=0}^{d} \delta_{t}^{j} S_{t}^{j}
$$

at time $t$ satisfies the SDE

$$
d V_{\delta}(t)=\sum_{j=0}^{d} \delta_{t}^{j} d S_{t}^{j}
$$

for all $t \in[0, T]$. The $j$ th component $\delta_{t}^{j}, j \in\{0,1, \ldots, d\}$, of the self-financing strategy $\delta$ expresses the number of units of the $j$ th primary security account held at time $t$ in the corresponding portfolio. Under a self-financing strategy no outflow or inflow of funds occurs for all changes in the value of the portfolio are due to gains from trade in the primary security accounts. Since we will only deal with self-financing portfolios and strategies, we omit the phrase "self-financing" in the sequel.

As is shown in Platen (2004b), to avoid portfolios with infinite growth potential, we need to assume that

$$
\sqrt{\tilde{\lambda}_{t}^{\ell-h_{1}}\left(\bar{z}_{t}\right)}>\theta_{t}^{\ell}
$$

for all $t \in[0, T]$ and $\ell \in\left\{h_{1}+1, \ldots, d\right\}$. 


\subsection{Growth Optimal Portfolio}

In a financial market model it is advantageous for derivative pricing and other risk management tasks to choose an appropriate reference unit, called a numeraire or benchmark. Under the benchmark approach, see Platen (2002, 2004a, 2004b, 2004d), we use the growth optimal portfolio (GOP) as benchmark. This is the self-financing portfolio $V_{\underline{\delta}}=\left\{V_{\underline{\delta}}(t), t \in[0, T]\right\}$ with the strategy $\underline{\delta}$ that achieves maximum expected logarithmic utility from terminal wealth. It has been shown in Platen (2004a, 2004c) that, under realistic assumptions, a global diversified portfolio is a good proxy for the GOP. This makes it a readily observable financial quantity, which can be used in various ways for risk management.

For the diffusion case without jumps, the SDE for the GOP is well known, see for instance, Long (1990) or Karatzas \& Shreve (1998). In the case with jumps, the derivation of the SDE for the GOP is more involved, using first order conditions for maximizing the drift of the logarithm of the portfolio. These lead to the SDE

$$
\begin{aligned}
d V_{\underline{\delta}}(t)=V_{\underline{\delta}}(t-) & \left(r_{t} d t+\sum_{i=1}^{h_{1}} \theta_{t}^{i}\left(\theta_{t}^{i} d t+d \tilde{v}_{t}^{i}\right)\right. \\
& \left.+\sum_{i=h_{1}+1}^{d} \frac{\theta_{t-}^{i}}{1-\frac{\theta_{t-}^{i}}{\sqrt{\tilde{\lambda}_{t-}^{i-h_{1}}\left(\bar{z}_{t-}\right)}}}\left(\theta_{t-}^{i} d t+d \tilde{m}_{t}^{i-h_{1}}\right)\right)
\end{aligned}
$$

for $t \in[0, T]$, with $V_{\underline{\delta}}(0)=1$, as demonstrated in Platen (2004b).

In what follows, we will call prices, which are expressed in units of the GOP, benchmarked prices. This means that the $j$ th benchmarked primary security account $\hat{S}^{(j)}=\left\{\hat{S}_{t}^{(j)}, t \in[0, T]\right\}$ has the value

$$
\hat{S}_{t}^{(j)}=\frac{S_{t}^{j}}{V_{\underline{\delta}}(t)}
$$

at time $t \in[0, T]$ for $j \in\{0,1, \ldots, d\}$. By (3.5), (3.13) and an application of the Itô formula the benchmarked primary security account $\hat{S}_{t}^{(j)}$ satisfies the SDE

$$
\begin{aligned}
d \hat{S}_{t}^{(j)}=\hat{S}_{t-}^{(j)} & \left(\sum_{i=1}^{h_{1}}\left(b_{t}^{j, i}-\theta_{t}^{i}\right) d \tilde{v}_{t}^{i}\right. \\
& \left.+\sum_{i=h_{1}+1}^{d}\left[b_{t-}^{j, i}\left(1-\frac{\theta_{t-}^{i}}{\sqrt{\tilde{\lambda}_{t-}^{i-h_{1}}\left(\bar{z}_{t-}\right)}}\right)-\theta_{t-}^{i}\right] d \tilde{m}_{t}^{i-h_{1}}\right)
\end{aligned}
$$

for $t \in[0, T]$ and $j \in\{0,1, \ldots, d\}$, see Platen (2004b). Similarly, by application of the Itô formula again, it can be shown that the value of any benchmarked 
portfolio $\hat{V}_{\delta}=\left\{\hat{V}_{\delta}(t), t \in[0, T]\right\}$, where

$$
\hat{V}_{\delta}(t)=\frac{V_{\delta}(t)}{V_{\underline{\delta}}(t)},
$$

satisfies the SDE

$$
\begin{aligned}
d \hat{V}_{\delta}(t)=\hat{V}_{\delta}(t-) & \left(\sum_{i=1}^{h_{1}}\left[\sum_{j=1}^{d} \frac{\delta_{t}^{j} \hat{S}_{t}^{(j)}}{\hat{V}_{\delta}(t)} b_{t}^{j, i}-\theta_{t}^{i}\right] d \tilde{v}_{t}^{i}\right. \\
& \left.+\sum_{i=h_{1}+1}^{d}\left[\left(\sum_{j=1}^{d} \frac{\delta_{t-}^{j} \hat{S}_{t-}^{(j)}}{\hat{V}_{\delta}(t-)} b_{t-}^{j, i}\right)\left(1-\frac{\theta_{t-}^{i}}{\sqrt{\tilde{\lambda}_{t-}^{i-h_{1}}\left(\bar{z}_{t-}\right)}}\right)-\theta_{t-}^{i}\right] d \tilde{m}_{t}^{i-h_{1}}\right)
\end{aligned}
$$

for $t \in[0, T]$, see Platen (2004b). Notice that all the coefficients in (3.17) are functions of observables.

Note furthermore that the $j$ th benchmarked primary security account $\hat{S}^{(j)}$, as well as all benchmarked portfolios, are driftless and are thus $\left(\tilde{\mathcal{A}}^{k}, P\right)$-local martingales. Therefore, any nonnegative benchmarked portfolio process is an $\left(\tilde{\mathcal{A}}^{k}, P\right)$ supermartingale. This means that it is impossible for a nonnegative portfolio to generate, with strictly positive probability, strictly positive wealth from zero initial capital. Consequently, the benchmark framework outlined here does not permit arbitrage in the sense of Platen (2004a).

In the literature there exist various mathematical definitions of arbitrage. The benchmark approach allows us to consider a more general class of models than is possible, for instance, under the no free lunch with vanishing risk concept developed in Delbaen \& Schachermayer $(1995,1998)$. The latter links no-arbitrage directly to the existence of an equivalent risk neutral measure. Such a measure need not exist in our framework. In the given benchmark framework, a free lunch with vanishing risk arises, for example, when the benchmarked savings account forms a strict $\left(\tilde{\mathcal{A}}^{k}, P\right)$-local martingale. Instances of this are described in Heath \& Platen (2002a, 2002c) and Breymann, Kelly \& Platen (2004).

\section{Fair Pricing of Derivatives}

\subsection{Derivative Price Processes as Martingales}

We emphasize that benchmarked security prices are, in general, not $\left(\tilde{\mathcal{A}}^{k}, P\right)$ martingales in our framework. However, we assume that any benchmarked derivative price process is fair, which means that it is an $\left(\tilde{\mathcal{A}}^{k}, P\right)$-martingale. By choosing the GOP as numeraire, the real world probability measure becomes the unique 
pricing measure for derivatives. We stress the fact that, even if there does not exist an equivalent martingale measure, it is still possible to operate with the GOP as numeraire and with the real world probability measure as pricing measure. This will be explained in what follows.

Above, we called a price process $V=\{V(t), t \in[0, T]\}$ fair if its benchmarked value $\hat{V}(t)=\frac{V(t)}{V_{\underline{\delta}}(t)}$ forms an $\left(\tilde{\mathcal{A}}^{k}, P\right)$-martingale under the available information represented by $\tilde{A}^{k}$. Recall that benchmarked nonnegative portfolios are $(\tilde{\mathcal{A}}, P)$ supermartingales and that benchmarked primary security accounts can be strict supermartingales. The fact that benchmarked derivative prices are fair puts buyers and sellers in comparable positions and generalizes the risk neutral approach, as we shall see below.

Note that $\tilde{\mathcal{A}}_{t}^{k}$ describes the information available at time $t$, whereas $\mathcal{A}_{t}$ is the complete information at time $t$ which determines the original model dynamics, including also the unobserved factors. Consequently, observed derivative prices may not be $(\underline{\mathcal{A}}, P)$-martingales, in general.

To provide an intuitive link between the general concept of fair pricing and standard risk neutral pricing as a special case, let us consider a candidate risk neutral probability measure $P^{k}$. This measure can be characterized by its RadonNikodym derivative process $\Lambda=\left\{\Lambda_{t}, t \in[0, T]\right\}$, which can be shown to be the benchmarked savings account

$$
\Lambda_{t}=\left.\frac{d P^{k}}{d P}\right|_{\mathcal{A}_{t}}=\frac{S_{t}^{(0)}}{V_{\underline{\delta}}(t)}=\hat{S}_{t}^{(0)}
$$

for $t \in[0, T]$. If $P^{k}$ is an equivalent risk neutral martingale measure, then the standard risk neutral pricing methodology and (4.1) yield for any portfolio $V_{\delta}$ the sequence of relations

$$
\begin{aligned}
V_{\delta}(t) & =S_{t}^{(0)} E^{P^{k}}\left(\frac{V_{\delta}(\tau)}{S_{\tau}^{(0)}} \mid \mathcal{A}_{t}\right)=S_{t}^{(0)} \frac{E\left(\Lambda_{\tau} \frac{V_{\delta}(\tau)}{S_{\tau}^{(0)}} \mid \mathcal{A}_{t}\right)}{E\left(\Lambda_{\tau} \mid \mathcal{A}_{t}\right)} \\
& =S_{t}^{(0)} \frac{E\left(\frac{V_{\delta}(\tau)}{V_{\underline{\delta}}(\tau)} \mid \mathcal{A}_{t}\right)}{\frac{S_{t}^{0}}{V_{\underline{\delta}}(t)}}=V_{\underline{\delta}}(t) E\left(\frac{V_{\delta}(\tau)}{V_{\underline{\delta}}(\tau)} \mid \mathcal{A}_{t}\right)
\end{aligned}
$$

for $\tau \in[0, T], t \in[0, \tau]$. Here $E^{P^{k}}$ denotes expectation with respect to $P^{k}$. From (4.2) it follows by (3.14) that

$$
\hat{V}_{\delta}(t)=E\left(\hat{V}_{\delta}(\tau) \mid \mathcal{A}_{t}\right)
$$

for $\tau \in[0, T], t \in[0, \tau]$ and $j \in\{0,1, \ldots, d\}$. This expresses the fact that $V_{\delta}$ is a fair price process and its discounted values form an $\left(\tilde{\mathcal{A}}, P^{k}\right)$-martingale in the standard risk neutral setup. 
It turns out that the measure $P^{k}$ above is the minimal equivalent martingale measure in the sense of Föllmer \& Schweizer (1991), under appropriate assumptions, see Platen $(2004 \mathrm{e})$. However, since we do not assume that $\Lambda$ is an $(\tilde{\mathcal{A}}, P)$ martingale and $P^{k}$ may not be equivalent to $P$, the first and second equalities in (4.2) may break down in our general benchmark framework.

Notice that at any instant $t \in[0, T]$, the value $V_{\underline{\delta}}(t)$ of the GOP represents that of a tradable portfolio, see (3.10), with the $\tilde{\mathcal{A}}^{k}$-predictable strategy $\underline{\delta}$. This portfolio invests in the primary security accounts, which are all observable. It follows, as already mentioned, that the GOP value $V_{\underline{\delta}}(t)$ is $\tilde{\mathcal{A}}_{t}^{k}$-measurable. This implies that the Radon-Nikodym derivative $\Lambda_{t}$, see (4.1), is observable at time $t$ and so, for the special case when $P^{k}$ is an equivalent risk neutral martingale measure, $\Lambda=\left\{\Lambda_{t}, t \in[0, T]\right\}$ is not only an $(\underline{\mathcal{A}}, P)$ - but also an $\left(\tilde{\mathcal{A}}^{k}, P\right)$-martingale. Relation (4.2) then holds, with $\tilde{\mathcal{A}}_{t}^{k}$ replacing $\mathcal{A}_{t}$. That is,

$$
V_{\delta}(t)=V_{\underline{\delta}}(t) E\left(\frac{V_{\delta}(\tau)}{V_{\underline{\delta}}(\tau)} \mid \tilde{\mathcal{A}}_{t}^{k}\right)
$$

for $t \in[0, T]$ and $j \in\{0,1, \ldots, d\}$. In this special case, it follows from (4.2) and the corresponding relation (4.4), with $\tilde{\mathcal{A}}_{t}^{k}$ instead of $\mathcal{A}_{t}$, that the triplets $\left(S^{(0)}, P^{k}, \mathcal{A}\right)$ and $\left(V_{\underline{\delta}}, P, \mathcal{A}\right)$ as well as $\left(S^{(0)}, P^{k}, \tilde{\mathcal{A}}^{k}\right)$ and $\left(V_{\underline{\delta}}, P, \tilde{\mathcal{A}}^{k}\right)$ define the same respective pricing systems. In our general situation this is not always the case. However, as we will show below, filtering and derivative pricing are still possible in a consistent manner under the benchmark approach, even when $P^{k}$ is not an equivalent martingale measure.

\subsection{Derivative Prices}

In what follows, denote by $\mathcal{T}_{t, T}$ the set of stopping times with values in $[t, T]$. For a given maturity date $\tau$, which is assumed to be an $\tilde{\mathcal{A}}^{k}$-stopping time, we consider a contingent claim $U\left(\tau, y_{\tau}\right)$ to be a nonnegative function of $\tau$ and the corresponding values of the observed factors $y_{\tau}$. We also assume that

$$
E\left(\frac{U\left(\tau, y_{\tau}\right)}{V_{\underline{\delta}}(\tau)} \mid \tilde{\mathcal{A}}_{t}^{k}\right)<\infty
$$

for all $\tau \in \mathcal{T}_{t, T}$ and $t \in[0, T]$. There is no point in letting the payoff function depend on any other factors, otherwise it would be indeterminable at time $\tau$ on the basis of available information.

Since, as mentioned in Section $4.1, V_{\underline{\delta}}(\tau)$ is $\tilde{\mathcal{A}}_{\tau}^{k}$-measurable, it can be considered as a function of $z_{s}$ for $s \leq \tau$. Furthermore, since $y_{\tau}$ is a subvector of $z_{\tau}$ and $z=$ $\left\{z_{t}, t \in[0, T]\right\}$ is a Markov process, we can define the process $u=\left\{u\left(t, z_{t}\right), t \in\right.$ $[0, T]\}$ as

$$
u\left(t, z_{t}\right)=E\left(\frac{V_{\underline{\delta}}(t)}{V_{\underline{\delta}}(\tau)} U\left(\tau, y_{\tau}\right) \mid \mathcal{A}_{t}\right)
$$


for $\tau \in \mathcal{T}_{t, T}$ and $t \in[0, T]$, which at time $t$ exploits the complete information characterized by the $\sigma$-algebra $\mathcal{A}_{t}$. Next, we consider

$$
\tilde{u}^{k}\left(t, \tilde{z}_{t}^{k}\right)=E\left(u\left(t, z_{t}\right) \mid \tilde{\mathcal{A}}_{t}^{k}\right)
$$

for $t \in[0, T]$. By Corollary 2.5, this can be computed on the basis of the filtering results of Section 2. Combining (4.6) with (4.7) and using the fact that $V_{\underline{\delta}}(t)$ is $\tilde{\mathcal{A}}_{t}^{k}$-measurable, we obtain

$$
\frac{\tilde{u}^{k}\left(t, \tilde{z}_{t}^{k}\right)}{V_{\underline{\delta}}(t)}=E\left(\frac{U\left(\tau, y_{\tau}\right)}{V_{\underline{\delta}}(\tau)} \mid \tilde{\mathcal{A}}_{t}^{k}\right)
$$

for $\tau \in \mathcal{T}_{t, T}$ and $t \in[0, T]$. This means that the benchmarked value $\frac{\tilde{u}^{k}\left(t, \tilde{z}_{t}^{k}\right)}{V_{\delta}(t)}$, stopped at the maturity $\tau$, forms a $\left(P, \tilde{\mathcal{A}}^{k}\right)$-martingale for $t \in[0, T]$. Obviously it is the only $\left(P, \tilde{\mathcal{A}}^{k}\right)$-martingale that coincides at time $\tau$ with $\frac{U\left(\tau, y_{\tau}\right)}{V_{\underline{\delta}}(\tau)}$. Thus, $\tilde{u}^{k}\left(t, \tilde{z}_{t}^{k}\right)$ is the fair price at time $t \leq \tau$ of the claim $U\left(\tau, y_{\tau}\right)$ on the basis of the information represented by $\tilde{\mathcal{A}}_{t}^{k}$.

The above concept of fair pricing, which can be applied generally, see Platen (2004a), extends the well-known concept of risk neutral pricing and avoids not only the assumption of the existence of an equivalent risk neutral measure, see Platen (2002), but also some delicate issues that arise from measure changes under different filtrations in filtering applications, see Bhar, Chiarella \& Runggaldier (2002). Therefore, under the benchmark approach, we enter not only a richer modeling world, but also avoid a number of technical issues that typically require special technical assumptions.

To illustrate again the special case when there exists an equivalent martingale measure $P^{k}$, we have, corresponding to (4.2) and using (4.6), that

$$
S_{t}^{(0)} E^{P^{k}}\left(\frac{U\left(\tau, y_{\tau}\right)}{S_{\tau}^{(0)}} \mid \mathcal{A}_{t}\right)=V_{\underline{\delta}}(t) E\left(\frac{U\left(\tau, y_{\tau}\right)}{V_{\underline{\delta}}(\tau)} \mid \mathcal{A}_{t}\right)=u\left(t, z_{t}\right)
$$

for $\tau \in \mathcal{T}_{t, T}$ and $t \in[0, T]$. In this special case, the same arguments as for relation (4.4), with $\tilde{\mathcal{A}}_{t}^{k}$ replacing $\mathcal{A}_{t}$, then lead to

$$
S_{t}^{(0)} E^{P^{k}}\left(\frac{U\left(\tau, y_{\tau}\right)}{S_{\tau}^{(0)}} \mid \tilde{\mathcal{A}}_{t}^{k}\right)=V_{\underline{\delta}}(t) E\left(\frac{U\left(\tau, y_{\tau}\right)}{V_{\underline{\delta}}(\tau)} \mid \tilde{\mathcal{A}}_{t}^{k}\right)=\tilde{u}^{k}\left(t, \tilde{z}_{t}^{k}\right)
$$

for $\tau \in \mathcal{T}_{t, T}$ and $t \in[0, T]$, using (4.6) and (4.7). Therefore, if there exists an equivalent martingale measure $P^{k}$, this implies that corresponding to (4.8), we have

$$
\frac{\tilde{u}^{k}\left(t, \tilde{z}_{t}^{k}\right)}{S_{t}^{(0)}}=E^{P^{k}}\left(\frac{U\left(\tau, y_{\tau}\right)}{S_{\tau}^{(0)}} \mid \tilde{\mathcal{A}}_{t}^{k}\right)=E^{P^{k}}\left(\frac{u\left(t, z_{t}\right)}{S_{t}^{(0)}} \mid \tilde{\mathcal{A}}_{t}^{k}\right)
$$

for $\tau \in \mathcal{T}_{t, T}$ and $t \in[0, T]$. This means that the discounted derivative price process $\frac{\tilde{u}^{k}\left(t, \tilde{z}_{t}^{k}\right)}{S_{t}^{(0)}}$, stopped at the maturity $\tau$, forms a $\left(P^{k}, \tilde{\mathcal{A}}^{k}\right)$-martingale, in this 
particular case. Furthermore, the last equality in (4.11) implies, just as in (4.7), that we also have

$$
\tilde{u}^{k}\left(t, \tilde{z}_{t}^{k}\right)=E^{P^{k}}\left(u\left(t, z_{t}\right) \mid \tilde{\mathcal{A}}_{t}^{k}\right)
$$

for $t \in[0, T]$, if an equivalent risk neutral martingale measure exists. We emphasize in this case that the expectations under different measures, in (4.7) and (4.12), lead to the same result, due to the fact that $\Lambda$ is a martingale, not only with respect to the filtration $\underline{\mathcal{A}}$, but also with respect to $\tilde{\mathcal{A}}^{k}$. In general, this does not hold under the benchmark framework.

Notice that if we did not consider fair pricing using the GOP and could use an equivalent risk neutral martingale measure, then we could try to perform the computations on the basis of formula (4.12). Although similar to the right hand side of (4.7), the right hand side of (4.12) is considerably more difficult to compute. This is due to the fact that in order to filter the process $z_{t}$ on the basis of the information contained in $\tilde{\mathcal{A}}_{t}^{k}$, we have to work under the real world probability measure $P$ in any case. Fortunately, since by (4.10) the quantity $\tilde{u}^{k}\left(t, \tilde{z}_{t}^{k}\right)$ computed according to (4.12) is the same as that in (4.7), we can perform the computations according to fair pricing, using (4.6) and (4.7). We thereby obtain the derivative price $\tilde{u}^{k}\left(t, \tilde{z}_{t}^{k}\right)$ under the information represented by $\tilde{\mathcal{A}}_{t}^{k}$. This shows that when it comes to actual computations, the real world measure plays a crucial and dominant role. Therefore, we suggest in this paper that filtering in finance should take place preferably under the real world measure. Most importantly, our approach is still applicable in the case where (4.12) fails to hold, which could be the result of wanting to use a realistic market model for which no equivalent martingale measure exists, for example.

Note that the expression in (4.7) perfectly matches the one for the filtered factor model given in (2.30). The actual computation of the conditional expectation in (4.7) is therefore equivalent to the solution of the filtering problem for the unobserved factors.

\subsection{Variance of Benchmarked Prices}

From a financial modeling point of view it is important to be able to model and understand different degrees of available information. This concerns issues such as insider trading, as well as the valuation of information. As already mentioned in Section 2.1, the degree of available information is indexed by the parameter $k$. A larger value for $k$ means that more factors are observed, thus providing more information in $\tilde{\mathcal{A}}^{k}$.

Let us now investigate the impact of varying degrees of information about the factors $z_{t}=\left(z_{t}^{1}, \ldots, z_{t}^{n}\right)^{\top}$ that underly our model dynamics, see $(2.2)-(2.3)$. We use the notation $\tilde{z}_{t}^{k}$, introduced in (2.13), for the vector of observables. We stress its dependence on $k$ and recall that, by (2.28), this process is Markovian. Consider 
now a contingent claim

$$
U\left(\tau, y_{\tau}\right)=U\left(\tau, y_{\tau}^{1}, y_{\tau}^{2}, \ldots, y_{\tau}^{r}\right)
$$

for some fixed $r \in\{1,2, \ldots, n-1\}$, where we assume that the number of observed factors that influence the claim equals $r$. For $k \in\{r, r+1, \ldots, n-1\}$, let $\tilde{u}^{k}\left(t, \tilde{z}_{t}^{k}\right)$ be the corresponding fair price at time $t$ under the information $\tilde{\mathcal{A}}_{t}^{k}$, as given by (4.7). Recall that, by $(4.7), \tilde{u}^{k}\left(t, \tilde{z}_{t}^{k}\right)$ is the conditional expectation, with respect to the real world measure, of $u\left(t, z_{t}\right)$ given $\tilde{\mathcal{A}}_{t}^{k}$. This implies that the corresponding conditional variance

$$
\operatorname{Var}_{t}^{k}(u)=E\left(\left(u\left(t, z_{t}\right)-\tilde{u}^{k}\left(t, \tilde{z}_{t}^{k}\right)\right)^{2} \mid \tilde{\mathcal{A}}_{t}^{k}\right)
$$

at time $t \in[0, T)$ is the minimal mean square difference between $u\left(t, z_{t}\right)$ and any $\tilde{\mathcal{A}}_{t}^{k}$-measurable random variable, conditional on $\tilde{\mathcal{A}}_{t}^{k}$. This conditional variance is computed under the real world measure. It would not make sense if computed under any other measure, since market participants are affected by the real difference between $u\left(t, z_{t}\right)$ and $\tilde{u}^{k}\left(t, \tilde{z}_{t}^{k}\right)$.

Note that the larger the value of $K$, the more information is available. Increasing $k$ should then naturally reduce the above conditional variance. The following practically relevant proposition quantifies this reduction in conditional variance. It can also be seen as a generalization of the celebrated Rao-Blackwell theorem, see Rao (1973), applied to filtering in incomplete markets under the benchmark approach.

Proposition 4.1 For $k \in\{r, r+1, \ldots, n-1\}$ and $m \in\{0,1, \ldots, n-k\}$, we have

$$
E\left(\operatorname{Var}_{t}^{k+m}(u) \mid \tilde{\mathcal{A}}_{t}^{k}\right)=\operatorname{Var}_{t}^{k}(u)-R_{t}^{k+m}
$$

where

$$
R_{t}^{k+m}=E\left(\left(\tilde{u}^{k+m}\left(t, \tilde{z}_{t}^{k+m}\right)-\tilde{u}^{k}\left(t, \tilde{z}_{t}^{k}\right)\right)^{2} \mid \tilde{\mathcal{A}}_{t}^{k}\right)
$$

for $t \in[0, T)$.

Proof: $\quad$ For $t \in[0, T)$ and $k \in\{r, r+1, \ldots, n-1\}$, we have

$$
\begin{aligned}
\left(u\left(t, z_{t}\right)-\tilde{u}^{k}\left(t, \tilde{z}_{t}^{k}\right)\right)^{2}= & \left(u\left(t, z_{t}\right)-\tilde{u}^{k+m}\left(t, \tilde{z}_{t}^{k+m}\right)\right)^{2}+\left(\tilde{u}^{k+m}\left(t, \tilde{z}_{t}^{k+m}\right)-\tilde{u}^{k}\left(t, \tilde{z}_{t}^{k}\right)\right)^{2} \\
& +2\left(u\left(t, z_{t}\right)-\tilde{u}^{k+m}\left(t, \tilde{z}_{t}^{k+m}\right)\right)\left(\tilde{u}^{k+m}\left(t, \tilde{z}_{t}^{k+m}\right)-\tilde{u}^{k}\left(t, \tilde{z}_{t}^{k}\right)\right) .
\end{aligned}
$$

By taking conditional expectations with respect to $\tilde{\mathcal{A}}_{t}^{k}$ on both sides of this equation, it follows that 


$$
\begin{gathered}
\operatorname{Var}_{t}^{k}(u)=E\left(\operatorname{Var}_{t}^{k+m}(u) \mid \tilde{\mathcal{A}}_{t}^{k}\right)+R_{t}^{k+m}+2 E\left(\left(\tilde{u}^{k+m}\left(t, \tilde{z}_{t}^{k+m}\right)-\tilde{u}^{k}\left(t, \tilde{z}_{t}^{k}\right)\right)\right. \\
\left.\cdot E\left(\left(u\left(t, z_{t}\right)-\tilde{u}^{k+m}\left(t, \tilde{z}_{t}^{k+m}\right)\right) \mid \tilde{\mathcal{A}}_{t}^{k+m}\right) \mid \tilde{\mathcal{A}}_{t}^{k}\right)
\end{gathered}
$$

Since the last term on the right hand side is equal to zero by definition, we obtain (4.15).

\section{Hedging Under Partial Observation}

To determine a hedging strategy in an incomplete market, we have to use a hedging criterion. It turns out that the fair pricing and hedging concept, developed in Platen (2004b), can be generalized to our situation. Already, when assuming the existence of an equivalent risk neutral martingale measure, it is known that there exist various hedging possibilities. In Platen (2004b) it has been pointed out that under the general benchmark approach, even in a complete market setting, there may exist different self-financing hedge portfolios that replicate a contingent claim. In general, under the benchmark approach, nonnegative benchmarked portfolios are $\left(\tilde{\mathcal{A}}^{k}, P\right)$-supermartingales, see $(3.17)$. The smallest possible supermartingale, which replicates the hedgable part of a contingent claim, is known to be a martingale. Therefore, among possible hedge portfolios, the fair portfolio process that replicates the hedgable part turns out to be special. It is the minimal portfolio replicating the hedgable part of the claim, because its benchmarked value is a martingale. To see this in more detail, let us introduce the benchmarked pricing function

$$
\hat{u}\left(t, \tilde{z}_{t}^{k}\right)=\frac{\tilde{u}^{k}\left(t, \tilde{z}_{t}^{k}\right)}{V_{\underline{\delta}}(t)}
$$

for $t \in[0, T]$. We introduce the differential operator

$$
L^{i} \hat{u}\left(t, \tilde{z}_{t}^{k}\right)=\sum_{\ell=1}^{k+q} \beta^{\ell, i}\left(t, \bar{z}_{t}^{1}, \ldots, \bar{z}_{t}^{k+q}\right) \frac{\partial \hat{u}\left(t, \tilde{z}_{t}^{k}\right)}{\partial \bar{z}^{\ell}}
$$

and the jump operator

$$
\begin{aligned}
\Delta_{\hat{u}}^{i}\left(t-, \tilde{z}_{t-}^{k}\right)= & \hat{u}\left(t, \bar{z}_{t-}^{1}+\gamma^{1, i}\left(t-, \bar{z}_{t-}^{1}, \ldots, \bar{z}_{t-}^{k+q}\right), \ldots,\right. \\
& \left.\bar{z}_{t-}^{k+q}+\gamma^{k+q, i}\left(t-, \bar{z}_{t-}, \ldots, \bar{z}_{t-}^{k+q}\right)\right) \\
& -\hat{u}\left(t-, \bar{z}_{t-}^{1}, \ldots, \bar{z}_{t-}^{k+q}\right)
\end{aligned}
$$

for $i \in\{1,2, \ldots, k\}$, where $\beta^{\ell, i}$ and $\gamma^{\ell, i}$ are as in (2.29). Here, we assume that the benchmarked pricing function $\hat{u}(\cdot, \cdot)$ in $(5.1)$ is differentiable with respect to time and twice differentiable with respect to the observables. Then we obtain 
from $(2.29),(5.2)$ and (5.3), by the Itô formula for the $\left(\tilde{\mathcal{A}}^{k}, P\right)$-martingale $\hat{u}=$ $\left\{\hat{u}\left(t, \tilde{z}_{t}^{k}\right), t \in[0, \tau]\right\}$, where $\tau \in \mathcal{T}_{t, T}$ and $t \in[0, T]$, the following representation for the benchmarked price of the contingent claim (4.13)

$$
\begin{aligned}
\frac{U\left(\tau, y_{\tau}\right)}{V_{\underline{\delta}}(\tau)} & =\hat{u}\left(\tau, \tilde{z}_{\tau}^{k}\right) \\
& =\hat{u}\left(t, \tilde{z}_{t}^{k}\right)+\hat{I}_{t, \tau}+\hat{R}_{t, \tau}
\end{aligned}
$$

Inspection of (3.4) reveals that (5.4) represents a decomposition of the benchmarked value of the claim into a hedgable part

$$
\hat{I}_{t, \tau}=\sum_{\ell=1}^{h_{1}} \int_{t}^{\tau} L^{\ell} \hat{u}\left(s, \tilde{z}_{s}^{k}\right) d \tilde{v}_{s}^{\ell}+\sum_{\ell=1}^{h_{2}} \int_{t}^{\tau} \Delta_{\hat{u}}^{\ell}\left(s-, \tilde{z}_{s-}^{k}\right) \sqrt{\tilde{\lambda}_{s-}^{\ell}\left(\bar{z}_{s-}\right)} d \tilde{m}_{s}^{\ell}
$$

and an unhedgable part

$$
\hat{R}_{t, \tau}=\sum_{\ell=h_{1}+1}^{k} \int_{t}^{\tau} L^{\ell} \hat{u}\left(s, \tilde{z}_{s}^{k}\right) d \tilde{v}_{s}^{\ell}+\sum_{\ell=h_{2}+1}^{k} \int_{t}^{\tau} \Delta_{\hat{u}}^{\ell}\left(s-, \tilde{z}_{s-}^{k}\right) \sqrt{\tilde{\lambda}_{s-}^{\ell}\left(\bar{z}_{s-}\right)} d \tilde{m}_{s}^{\ell} .
$$

Note that $(5.4)$ is an $\left(\tilde{\mathcal{A}}^{k}, P\right)$-martingale representation for the benchmarked contingent claim. Obviously, there is no way to hedge fluctuations that arise in the unhedgable part with tradable securities.

One can now search for a fair benchmarked portfolio process $\hat{V}_{\delta_{U}}$, with selffinancing hedging strategy $\delta_{U}=\left\{\delta_{U}(t)=\left(\delta_{U}^{0}(t), \delta_{U}^{1}(t), \ldots, \delta_{U}^{d}(t)\right)^{\top}, t \in[0, \tau]\right\}$, that replicates the hedgable exposure $\hat{I}_{t, \tau}$. To do this, we compare the SDE (3.17) for $\hat{V}_{\delta_{U}}(t)$ with that for the hedgable part $\hat{I}_{t, \tau}$ of the claim, see (5.5). Let us write

$$
\pi_{\delta_{U}}^{j}(t)=\frac{\delta_{U}^{j}(t) \hat{S}_{t}^{(j)}}{\hat{V}_{\delta_{U}}(t)}
$$

for the proportion of the value of the hedge portfolio to be invested in the $j$ th primary security account at time $t \in[0, \tau]$ for $j \in\{0,1, \ldots, d\}$. By the above comparison, it follows that one needs to satisfy the equation

$$
\sum_{j=1}^{d} \pi_{\delta_{U}}^{j}(t) b_{t}^{j, i}-\theta_{t}^{i}=\frac{L^{i} \hat{u}\left(t, \tilde{z}_{t}^{k}\right)}{\hat{V}_{\delta_{U}}(t)}
$$

for $i \in\left\{1,2, \ldots, h_{1}\right\}$, as well as the equation

$$
\left(\sum_{j=1}^{d} \pi_{\delta_{U}}^{j}(t-) b_{t-}^{j, i}\right)\left(1-\frac{\theta_{t-}^{i}}{\sqrt{\tilde{\lambda}_{t-}^{i-h_{1}}\left(\bar{z}_{t-}\right)}}\right)-\theta_{t-}^{i}=\frac{\Delta_{\hat{u}}^{i}\left(t-, \tilde{z}_{t-}^{k}\right)}{\hat{V}_{\delta_{U}}(t-)}
$$


for $i \in\left\{1, \ldots, h_{2}\right\}$. Given the vectors $e_{U}(t)=\left(e_{U}^{1}(t), \ldots, e_{U}^{d}(t)\right)^{\top}$, where

$$
e_{U}^{\ell}(t-)=\left\{\begin{array}{cl}
\frac{L^{\ell} \hat{u}\left(t-, \tilde{z}_{t-}^{k}\right)}{\hat{V}_{\delta_{U}}(t-)}+\theta_{t-}^{\ell} & \text { for } \quad \ell \in\left\{1,2, \ldots, h_{1}\right\} \\
\frac{\sqrt{\tilde{\hat{\lambda}}_{t-}^{i-h_{1}}\left(\bar{z}_{t-}\right)}\left(\frac{\Delta_{\hat{u}}^{i}\left(t-, \tilde{z}_{t-}^{k}\right)}{\hat{V}_{\delta_{U}}^{(t-)}}+\theta_{t-}^{i}\right)}{\sqrt{\tilde{\hat{\lambda}}_{t-}^{i-h_{1}}\left(\bar{z}_{t-}\right)}-\theta_{t-}^{i}} & \text { for } \quad \ell=h_{1}+i \in\left\{h_{1}+1, \ldots, d\right\}
\end{array}\right.
$$

and $\pi_{\delta_{U}}(t)=\left(\pi_{\delta_{U}}^{1}(t), \ldots, \pi_{\delta_{U}}^{d}(t)\right)^{\top}$, equations (5.8) and (5.9) yield the condition

$$
e_{U}(t-)=\left(\pi_{\delta_{U}}^{\top}(t-) b_{t-}\right)^{\top}
$$

Consequently, we can formulate the following result.

Proposition 5.1 The hedgable part of the contingent claim $U$ can be replicated by the portfolio $V_{\delta_{U}}$ whose proportions are

$$
\pi_{\delta_{U}}(t)=\left(e_{U}(t)^{\top} b_{t}^{-1}\right)^{\top}
$$

for $t \in[0, T]$ and $\tau \in \mathcal{T}_{t, T}$.

Notice that the elements of the vector $e_{U}(t)$ and of the matrix $b_{t}$ are functions of observables. Since part of the latter are the results of filtering, this shows the usefulness of filtering for hedging under partial observation.

Note furthermore that the driving martingales in the unhedgable part $\hat{R}_{t, \tau}$, see (5.6), are orthogonal to those that drive the primary security accounts and thus orthogonal to the hedgable part $\hat{I}_{t, \tau}$, see (5.5). The above fair hedging strategy minimizes the quadratic variation of the resulting benchmarked profit and loss process under the real world measure. In Platen (2004e) it is derived as fluctuation minimization hedge. Obviously, to perform the fair hedge, the corresponding initial capital at time $t$ needs to equal the fair price $\tilde{u}^{k}\left(t, \tilde{z}_{t}^{k}\right)$, see (4.7), of the contingent claim.

In the special case when an equivalent risk neutral martingale measure exists, the resulting hedging strategy equals the local risk minimizing strategy in the sense of Föllmer \& Schweizer (1991) and the pricing measure is the minimal equivalent martingale measure, see Hofmann, Platen \& Schweizer (1992). The martingale representation (5.4) is in this special case the corresponding benchmarked version of the Föllmer-Schweizer decomposition. We want to point out that, to the best of our knowledge, the literature concerning local risk minimization under partial information, see Schweizer (1994), Fischer, Platen \& Runggaldier (1999) and Frey \& Runggaldier (1999), assumes from the outset that everything is defined under a risk neutral measure. This unpleasant and restrictive assumption is avoided in our approach, which makes filtering more practicable. 


\section{Conclusions}

We constructed a jump-diffusion financial market model with hidden variables and specified as benchmark the growth optimal portfolio. The random driving processes are Wiener and Poisson jump processes. For this incomplete market model with partial observation a consistent price system has been established without assuming the existence of an equivalent risk neutral martingale measure. Benchmarked fair derivative prices are obtained as martingales under the real world probability measure. Filtering has been described as an essential method for implementing fair pricing and hedging under partial information in the given incomplete market. The reduction of the conditional variance of fair derivative prices, when the available information increases, is quantified via a generalization of the Rao-Blackwell theorem.

\section{A Appendix}

\section{Proof of Proposition 2.3}

Denote by $y^{c}$ the continuous part of the observation process $y$, that is

$$
y_{t}^{c}=y_{t}-\sum_{\tau_{j} \leq t} G_{\tau_{j}-}\left(y_{\tau_{j}-}\right) \Delta N_{\tau_{j}}
$$

where the $\tau_{j}$ denote the jump times of $N=\left\{N_{t}, t \in[0, T]\right\}$ and $\Delta N_{\tau_{j}}=N_{\tau_{j}}-$ $N_{\tau_{j}-}$ is the vector $\left(\Delta N_{\tau_{j}-}^{1}, \ldots, \Delta N_{\tau_{j}-}^{k}\right)^{\top}$. Let us now define the $k$-dimensional $\tilde{\mathcal{A}}^{k}$-adapted process $\tilde{v}=\left\{\tilde{v}_{t}, t \in[0, T]\right\}$ by

$$
B_{t}\left(y_{t}\right) d \tilde{v}_{t}=d y_{t}^{c}-\tilde{A}_{t}\left(\tilde{z}_{t}^{k}\right) d t .
$$

From (2.3), (A.1) and (A.2) it follows that

$$
d \tilde{v}_{t}=d v_{t}+B_{t}\left(y_{t}\right)^{-1}\left[A_{t}\left(z_{t}\right)-\tilde{A}_{t}\left(\tilde{z}_{t}^{k}\right)\right] d t .
$$

From this we find, by the multi-variate Itô formula with $\nu \in \Re^{k}$ a row vector and $\imath$ the imaginary unit, that

$$
\begin{aligned}
\exp \left[\imath \nu\left(\tilde{v}_{t}-\tilde{v}_{s}\right)\right]= & 1+\imath \nu \int_{s}^{t} \exp \left[\imath \nu\left(\tilde{v}_{u}-\tilde{v}_{s}\right)\right] d v_{u} \\
& +\imath \nu \int_{s}^{t} \exp \left[\imath \nu\left(\tilde{v}_{u}-\tilde{v}_{s}\right)\right] B_{u}^{-1}\left(y_{u}\right)\left(A_{u}\left(z_{u}\right)-\tilde{A}_{u}\left(\tilde{z}_{u}^{k}\right)\right) d u \\
& -\frac{\nu \nu^{\top}}{2} \int_{s}^{t} \exp \left[\imath \nu\left(\tilde{v}_{u}-\tilde{v}_{s}\right)\right] d u
\end{aligned}
$$


Recalling that $v$ is an $\tilde{\mathcal{A}}^{k}$-measurable Wiener process, notice that

$$
E\left(\int_{s}^{t} \exp \left[\imath \nu\left(\tilde{v}_{u}-\tilde{v}_{s}\right)\right] d v_{u} \mid \tilde{\mathcal{A}}_{s}^{k}\right)=0
$$

and that, by our assumptions, the boundedness of $\exp \left[\imath \nu\left(\tilde{v}_{u}-\tilde{v}_{s}\right)\right]$ and the $\tilde{\mathcal{A}}_{t}^{k}$ measurability of $B_{u}^{-1}\left(y_{n}\right)$

$$
\begin{aligned}
E\left(\int_{s}^{t} \exp \left[\imath \nu\left(\tilde{v}_{u}-\tilde{v}_{s}\right)\right] B_{u}^{-1}\left(y_{u}\right)\left(A_{u}\left(z_{u}\right)-\tilde{A}_{u}\left(\tilde{z}_{u}^{k}\right)\right) d u \mid \tilde{\mathcal{A}}_{s}^{k}\right) & = \\
E\left(\int_{s}^{t} \exp \left[\imath \nu\left(\tilde{v}_{u}-\tilde{v}_{s}\right)\right] B_{u}^{-1}\left(y_{u}\right) E\left(\left(A_{u}\left(z_{u}\right)-\tilde{A}_{u}\left(\tilde{z}_{u}^{k}\right)\right) \mid \tilde{\mathcal{A}}_{u}^{k}\right) d u \mid \tilde{\mathcal{A}}_{s}^{k}\right) & =0 .
\end{aligned}
$$

Taking conditional expectations on the left and the right hand sides of (A.4) we end up with the equation

$$
E\left(\exp \left(\imath \nu\left[\left(\tilde{v}_{t}-\tilde{v}_{s}\right)\right]\right) \mid \tilde{\mathcal{A}}_{s}^{k}\right)=1-\frac{\nu \nu^{\prime}}{2} \int_{s}^{t} E\left(\exp \left[\imath \nu\left(\tilde{v}_{u}-\tilde{v}_{s}\right)\right] \mid \tilde{\mathcal{A}}_{s}^{k}\right) d u,
$$

which has the solution

$$
E\left(\exp \left[\imath \nu\left(\tilde{v}_{t}-\tilde{v}_{s}\right)\right] \mid \tilde{\mathcal{A}}_{s}^{k}\right)=\exp \left[-\frac{\nu \nu^{\top}}{2}(t-s)\right]
$$

for $0 \leq s \leq t \leq T$. We can conclude that $\left(\tilde{v}_{t}-\tilde{v}_{s}\right)$ is a $k$-dimensional vector of independent $\tilde{\mathcal{A}}_{t}^{k}$-measurable Gaussian random variables, each with variance $(t-s)$ and independent of $\tilde{\mathcal{A}}_{s}^{k}$. By Levy's theorem, $\tilde{v}$ is thus a $k$-dimensional $\tilde{\mathcal{A}}^{k}$-adapted standard Wiener process.

\section{Acknowledgement}

The authors would like to thank two anonymous referees, the Editor of APFM and Hardy Hulley for valuable suggestions on the subject of the paper.

\section{References}

Bajeux-Besnainou, I. \& R. Portait (1997). The numeraire portfolio: A new perspective on financial theory. The European Journal of Finance 3, 291309.

Bhar, R., C. Chiarella, \& W. Runggaldier (2002). Estimation in models of the instantaneous short term interest rate by use of a dynamic Baysian algorithm. In K. Sandmann and P. Schoenbucher (Eds.), Advances in Finance and Stochastics - Essays in Honour of Dieter Sondermann, pp. 177-195. Springer. 
Bhar, R., C. Chiarella, \& W. Runggaldier (2004). Inferring the forward looking equity risk premium from derivative prices. Studies in Nonlinear Dynamics \& Econometrics 8(1). Article 3, http://www.bepress.com/snde/vol8/iss1/art3.

Breymann, W., L. Kelly, \& E. Platen (2004). Intraday empirical analysis and modeling of diversified world stock indices. Technical report, University of Technology, Sydney. QFRC Research Paper 125.

Chiarella, C., S. Pasquali, \& W. Runggaldier (2001). On filtering in Markovian term structure models (an approximation approach). Adv. in Appl. Probab. 33(4), 794-809.

Delbaen, F. \& W. Schachermayer (1995). The no-arbitrage property under a change of numeraire. Stochastics Stochastics Rep. 53, 213-226.

Delbaen, F. \& W. Schachermayer (1998). The fundamental theorem of asset pricing for unbounded stochastic processes. Math. Ann 312, 215-250.

Elliott, R. J., P. Fischer, \& E. Platen (1999). Hidden Markov filtering for a mean reverting interest rate model. In 38th IEEE CDC meeting in Phoenix, pp. 2782-2787. IEEE Control Systems Society.

Elliott, R. J. \& E. Platen (2001). Hidden Markov chain filtering for generalised Bessel processes. In Stochastics in Finite and Infinite Dimensions, pp. 123143. Birkhäuser.

Elliott, R. J. \& J. van der Hoek (1997). An application of hidden Markov models to asset allocation problems. Finance Stoch. 1, 229-238.

Fischer, P. \& E. Platen (1999). Applications of the balanced method to stochastic differential equations in filtering. Monte Carlo Methods Appl. 5(1), 1938.

Fischer, P., E. Platen, \& W. Runggaldier (1999). Risk minimizing hedging strategies under partial observation. In Seminar on Stochastic Analysis, Random Fields and Applications, Volume 45 of Progr. Probab., pp. 175188. Birkhäuser.

Föllmer, H. \& M. Schweizer (1991). Hedging of contingent claims under incomplete information. In M. Davis and R. Elliott (Eds.), Applied Stochastic Analysis, Volume 5 of Stochastics Monogr., pp. 389-414. Gordon and Breach, London/New York.

Frey, R. \& W. J. Runggaldier (1999). Risk minimizing hedging strategies under restricted information: The case of stochastic volatility models observable only at random discrete times. Math. Methods Oper. Res. 50, 339-350.

Frey, R. \& W. J. Runggaldier (2001). A nonlinear filtering approach to volatility estimation with a view towards high frequency data. Int. J. Theor. Appl. Finance 4(2), 199-210. 
Gombani, A. \& W. J. Runggaldier (2001). A filtering approach to pricing in multifactor term structure models. Int. J. Theor. Appl. Finance 4(2), 303320.

Heath, D. \& E. Platen (2002a). Consistent pricing and hedging for a modified constant elasticity of variance model. Quant. Finance. 2(6), 459-467.

Heath, D. \& E. Platen (2002b). Perfect hedging of index derivatives under a minimal market model. Int. J. Theor. Appl. Finance 5(7), 757-774.

Heath, D. \& E. Platen (2002c). Pricing and hedging of index derivatives under an alternative asset price model with endogenous stochastic volatility. In J. Yong (Ed.), Recent Developments in Mathematical Finance, pp. 117-126. World Scientific.

Hofmann, N., E. Platen, \& M. Schweizer (1992). Option pricing under incompleteness and stochastic volatility. Math. Finance 2(3), 153-187.

Karatzas, I. \& S. E. Shreve (1998). Methods of Mathematical Finance, Volume 39 of Appl. Math. Springer.

Korn, R. \& M. Schäl (1999). On value preserving and growth-optimal portfolios. Math. Methods Oper. Res. 50(2), 189-218.

Landen, C. (2000). Bond pricing in a hidden Markov model of the short rate. Finance Stoch. 4, 371-385.

Liptser, R. \& A. Shiryaev (1977). Statistics of Random Processes: I. General Theory, Volume 5 of Appl. Math. Springer.

Long, J. B. (1990). The numeraire portfolio. J. Financial Economics 26, 29-69.

Platen, E. (2002). Arbitrage in continuous complete markets. Adv. in Appl. Probab. 34(3), 540-558.

Platen, E. (2004a). A benchmark framework for risk management. In Stochastic Processes and Applications to Mathematical Finance, pp. 305-335. Proceedings of the Ritsumeikan Intern. Symposium: World Scientific.

Platen, E. (2004b). A class of complete benchmark models with intensity based jumps. J. Appl. Probab. 41, 19-34.

Platen, E. (2004c). Diversified portfolios with jumps in a benchmark framework. Technical report, University of Technology, Sydney. QFRC Research Paper 129.

Platen, E. (2004d). Modeling the volatility and expected value of a diversified world index. Int. J. Theor. Appl. Finance 7(4), 511-529.

Platen, E. (2004e). Pricing and hedging for incomplete jump diffusion benchmark models. In Mathematics of Finance, Proceedings of an AMS-IMS-SIAM Joint Summer Research Conf on Mathematics of Finance, June 2003, Snowbird, Utah, Volume 351 of Contemporary Mathematics, pp. 287-301. American Mathematical Society. 
Platen, E. \& G. Stahl (2003). A structure for general and specific market risk. Computational Statistics 18(3), 355 - 373.

Protter, P. (1990). Stochastic Integration and Differential Equations. Springer.

Rao, C. R. (1973). Linear Statistical Inference and Its Applications (2nd ed.). Wiley, New York.

Schweizer, M. (1994). Risk minimizing hedging strategies under restricted information. Math. Finance 4(4), 327-342. 